\title{
Association of S. haematobium Infection Morbidity and Severity on Co- infections status in Pre-school Age Children Living in a Rural Endemic Area in Zimbabwe
}

\section{Tariro Lavender Mduluza-Jokonya ( $\nabla$ tljokonya@gmail.com )}

University of kwaZulu Natal https://orcid.org/0000-0002-1219-6464

Thajasvarie Naicker

University of KwaZulu-Natal College of Health Sciences

Luxwell Jokonya

University of KwaZulu-Natal College of Health Sciences

\section{Herald Midzi}

University of KwaZulu-Natal College of Health Sciences

Arthur Vengesai

University of KwaZulu-Natal College of Health Sciences

Maritha Kasambala

University of Kwazulu-Natal

\section{Emelia Choto}

University of KwaZulu-Natal College of Health Sciences

\section{Simbarashe Rusakaniko}

University of Zimbabwe College of Health Sciences

\section{Elopy Sibanda}

Twin Palm Clinic Harare

\section{Francisca Mutapi}

The University of Edinburgh Institute of Immunology and Infection Research

\section{Takafira Mduluza}

University of Zimbabwe Faculty of Science

\section{Research article}

Keywords: schistosomiasis, $<5$ mortality rate, communicable diseases, acute respiratory infection, fever of unknown origin, PSAC

Posted Date: March 31st, 2020

DOl: https://doi.org/10.21203/rs.3.rs-19172/v1

License: (1) This work is licensed under a Creative Commons Attribution 4.0 International License. Read Full License 


\section{Abstract}

Background: Individuals living in S. haematobium endemic areas are often at risk of having other communicable diseases, simultaneously. This usually creates diagnostic difficulties leading to misdiagnosis and overlooking schistosomiasis infection. In this study we investigated the prevalence and effects of coinfections in pre-school age children.

Methodology : About 465 Preschool age children were clinically examined for the following top morbidity conditions: respiratory tract infections, dermatophytosis, malaria and fever of unknown origin. S. haematobium infection was diagnosed by urine filtration and the children were screened for other communicable infections common in rural areas.

Results : Prevalence of S. haematobium was 35\% (145). A positive relationship between S. haematobium prevalence and under-5 mortality rate in Zimbabwean provinces was demonstrated. The odds of co-infections observed for S. haematobium were: upper respiratory tract infection AOR = 1.98 (95\% Cl 1.657 to 2.48 ), dermatophytosis $\mathrm{AOR}=5.10$ ( $95 \% \mathrm{Cl} 2.99$ to 8.72 ), fever of unknown origin $\mathrm{AOR}=9.07$ ( $95 \% \mathrm{Cl} 5.70$ to 14.44$)$ and malaria $\mathrm{AOR}=0.91(95 \% \mathrm{Cl} 0.54$ to 1.54$)$. The risk ratio of having $\mathrm{S}$. haematobium and co-infections in children who had fever of unknown origin $138 \%$, dermatophytosis $38 \%$, Upper respiratory tract infection $1 \%$ increase risk and malaria had a $2 \%$ reduced risk. Odds of having severe sequelae following the above conditions were: severe pneumonia $\mathrm{AOR}=8.41(95 \% \mathrm{Cl} 3.09-22.93)$, complicated malaria $\mathrm{AOR}=7.09(95 \% \mathrm{Cl} 1.51-33.39)$, severe and persistent dermatophytosis $\mathrm{AOR}=20.3(95 \% \mathrm{Cl} 4.78-83.2)$ and seizures $\mathrm{AOR}=1.62(95 \% \mathrm{Cl} 1.56-4.73)$.

Conclusion : This study is novel as it identifies a possible causal relationship between S. haematobium infection and top morbidity conditions in children under five years. There is need to alert policy makers so as to initiate early treatment of schistosomiasis in pre-school age children.

\section{Introduction}

Human schistosomiasis is a parasitic disease caused by blood flukes called trematode worms of the genus Schistosoma, predominantly affecting people in low- and middle-income countries(1). An estimated 206.4 million people in 78 countries required treatment for schistosomiasis in 2016(2,3). In sub-Saharan Africa alone, about 52 endemic countries reported moderate to high prevalence (4). Individuals living in schistosomiasis-endemic areas are often at risk to several pathogens simultaneously (5). These coinfections could arise due to chance because of host susceptibility or co-circulation of various disease agents (6). Alternatively, schistosomiasis may increase or decrease the risk for another infection (7). Studies on co-infection in adults have shown that schistosomiasis co-infection hinders diagnosis and treatment of other communicable diseases $[8,10]$. To date, however, little focus has been given to pre-school age children (PSAC), who were regarded as a low risk group. However, recent studies suggest that they may have similar risk to adults (11). The prevalence and relationship of schistosomiasis to coinfections in PSAC has not yet been described.

The World Health Organization (WHO) Sustainable Developmental Goal (SDG) 3 aims to reduce under-5 mortality to at least as low as 25 per 1,000 live births in every country by 2030 (4). In Zimbabwe, the top causes of morbidity and mortality in PSAC include acute respiratory tract infections (ARI), malaria, diarrhoea, fever and skin diseases(12)(13). In this study we investigated the prevalence and extent of morbidity associated with S. haematobium coinfections in children under the age of five years in an endemic district of Zimbabwe.

\section{Methodology}

\section{Study site and design}

The study was conducted in Shamva District in Mashonaland Central Province of Zimbabwe, with the highest prevalence of schistosomiasis in Zimbabwe at 62.3\%. Children were recruited from 19 different villages in Shamva district and Shamva district hospital. All children were assessed for schistosomiasis, upper respiratory tract infections, dermatophytosis and malaria. Clinical screening was done by experienced clinician and laboratory work was done by experienced laboratory scientist.

\section{Study inclusion criteria}

Participants recruited into the study were residents of the Shamva district in Zimbabwe. The PSAC were aged between 1 to 5 years and met the following inclusion criteria: 1. Be lifelong residents of the study area 2. Had no previous anti-helminthic treatment exposure 3. Parental/guardian consent to participate 4. Be negative for Schistosoma mansoni and geohelminths 5. Be negative for the ToRCHeS (toxoplasmosis, rubella, cytomegalovirus, hepatitis and syphilis) screen 6. Be HIV negative 8. Have a widal TO ratio <1:160 9. Mantoux test reaction <5mm 10. Had a normal nutrition status

\section{Ethical statement}

Ethical approval was obtained from Medical Research Council of Zimbabwe (MRCZ/A/2435). Gatekeeper approval was obtained from the Provincial and District Medical Directors and Community Leaders. Written informed consent was obtained from the parents or guardians of the 
children. All participants with confirmed infections were offered treatment.

\section{Data collection}

A questionnaire was administered and medical records assessed for those who were admitted. Information was extracted from the demographic and health survey- Zimbabwe statistics (13). The coinfections were selected from top morbidity causes in children under-five years old in

Zimbabwe (12).

\section{Clinical examinations}

The clinical examinations were conducted on PSAC $(n=415)$ by two medical practitioners independent of each other according to a protocol (Figure 1)

\section{$\underline{\text { S. haematobium infection diagnosis }}$}

Urine samples collected were examined for macrohematuria using the Uristix reagent strips (Uripath, Plasmatec, UK) dipped into fresh, well-mixed urine for $40 \mathrm{sec}$ and the test area was compared with a standard colour chart as per manufacturer's instructions. The parasitology team conducted parasitology examination and results were recorded separately, not accessed by the clinical team. Approximately 50 ml of urine sample was collected from each participant on three consecutive days. The samples were collected between 10 am and 2 pm and processed within 2 hours of collection by urine filtration method and were examined using microscopy for $S$. haematobium eggs detection. The number of eggs were reported per $10 \mathrm{ml}$ of urine.

\section{Diagnosis of other infections}

\section{Upper Respiratory Tract Infection}

URTI was diagnosed on clinical signs and symptoms after excluding allergy and influenza (14-16). Severe pneumonia was defined as per WHO guidelines $(17,18)$.

\section{Fever of unknown origin (FUO)}

FUO was defined as children who within the past six months had been admitted with a temperature of $38.5^{\circ} \mathrm{C}$ and no other diagnosis found after blood, urine and stool cultures as represented from their medical records (19). Seizures were described as change in movement, attention or loss of consciousness in a child diagnosed with FUO, with no family history of febrile seizures (20).

\section{Malaria}

Thick and thin blood film slides were stained using the geimsa stain and examined for malaria parasites using microscopy, as previously described(21). Complicated malaria was described as per WHO guideline for severe malaria $(18,22)$.

\section{Dermatophytosis}

Skin scraps were collected from individuals with signs of dermatophytosis, examined on a warmed potassium hydroxide treated slide for microscopy(23). Severe dermatophytosis was described as ringworms covering greater than $20 \%$ surface area using the paediatric burns chart (24).

\section{Statistical method}

Data analysis was performed using STATA version 15. The statistical methods applied were descriptive statistics providing relative risk and regression analysis for odds ratio. Results were reported as adjusted ORs (AORs) and risk ratios (RR) with 95\% confidence interval (CI), along with the test for significance, as previously described (25). Infection intensity for $S$. haematobium was defined as the arithmetic mean egg count/10ml of at least two urine samples collected on three consecutive days.

\section{Results}

Screening involved 465 PSAC aged one to five years from the Shamva district, 415 of whom met the eligibility criteria and consented to be part of the study. The sex ratio was equal, and the mean age was 3.39 years. Those with malaria had $P$. falciparum as the parasite.

The prevalence of S. haematobium was 35.1\% (145) While among study participants, 40\% (229) presented with a URTI , 45\% (188) with FUO and $18 \%$ (75) with dermatophytosis and malaria as well (Table 1). The prevalence of co-infections with S. haematobium was: URTI 35\% (80), malaria $33.3 \%$ (25), FUO 55\% (91) and dermatophytosis 67\% (50). 
In univariate analysis, the following associations with schistosomiasis infection had significant odds ratio(OR): URTI OR=1.98 (95\% Cl 1.657 to 2.48), dermatophytosis OR=5.10 ( $95 \% \mathrm{Cl} 2.99$ to 8.72) and FUO OR=9.07 ( $95 \% \mathrm{Cl} 5.70$ to 14.44 )(Table 2). In multivariable analysis, after adjusting for age, sex ova in urine and infection status, the following were independently associated adjusted odds ratio (AOR): dermatophytosis $\mathrm{AOR}=4.79$ ( $95 \% \mathrm{Cl} 2.78$ to 8.25$)$ and FUO AOR=10.63 (95\% Cl 6.48 to 17.45$)$.

We demonstrated a positive relationship between S. haematobium infection with child mortality and under-5 mortality in Zimbabwean Provinces (Figure2). Provinces with an increased schistosomiasis prevalence (Manicaland, Mashonaland East, West and Central) all showed an increased under-five and child mortality rate. Whereas Matebeleland North and Bulawayo had both a decrease in S. haematobium infection prevalence and mortality ratios.

\section{Severity of morbidity associated with co-infections}

PSAC with schistosomiasis and URTI had an eight-fold higher odds of pneumonia than children with URTI alone AOR=7.90 ( $95 \% \mathrm{Cl} 2.76$ to 27.5 ), $\mathrm{p}=0.008$ (Table 3). Children with schistosomiasis and malaria had a 7-times greater odds of complicated malaria $\mathrm{AOR}=7.09$ (95\% $\mathrm{Cl} 1.51$ to 33.39), $p=0.005$. Children with dermatophytosis had a 20 -fold higher odds of severe dermatophytosis $\mathrm{AOR}=20.3(95 \% \mathrm{Cl} 4.78$ to $83.2 ; \mathrm{p}=<0.001)$ compared with children who did not have schistosomiasis. Among children with FUO, those who also had schistosomiasis coinfection had twice the chance of seizures AOR=1.62 (95\% Cl 1.56 to 4.73$)$.

\section{Discussion}

Children growing up in resource limited rural areas have high exposure to schistosomiasis infection and a high likelihood of associated coinfections with diseases prevalent in poor communities. Considering URTI, dermatophytosis and FUO; a strong positive association between schistosomiasis and URTI; dermatophytosis and FUO were observed. The trends revealed an association between schistosomiasis and under-5 mortality rate from the national data provided by the Ministry of Health in Zimbabwe. There was a negative association between schistosomiasis and malaria, though participants with the two as co-infections had a greater likelihood of presenting with complicated malaria. Similarly, participants with URTI, FOU and dermatophytosis as coinfections had a higher likelihood of having severe sequelae of the diseases.

A positive association on comparing schistosomiasis prevalence with under-5 and child mortality rate in different provinces of Zimbabwe was demonstrated. The provinces that had a high schistosomiasis rate also had high mortality rate(12,13). This made us wonder if schistosomiasis co-infections were possibly worsening the disease courses as we also found that in co-infections there was a greater chance of the disease course turning out to be severe (12). It is necessary to explore the effects of schistosomiasis and other diseases co-infections in all the top morbidity and mortality causes in PSAC. Early schistosomiasis treatment in PSAC has the potential to lower the under-five mortality rate, by reducing the incidence of severe sequelae of the top morbidity conditions in this age group which are also top causes for mortality in this age group. This should be shared with policy makers in low and middle income countries.

Children infected with S. haematobium had a 20 -fold higher odds of severe dermatophytosis, after adjusting for other clinical conditions. $S$. haematobium infection had a $38 \%$ increased risk of getting dermatophytosis. There is no previous documentation between $S$. haematobium and dermatophytosis. In literature extensive dermatophytosis was noted in a case report involving S. mansoni (26). It is postulated that S.mansoni exacerbated dermatophytosis by lowering immunity due to the liver involvement or by suppression of the T-helper 1 system which is involved in suppressing fungal infections $(27,28)$. Dermatophytosis cause great morbidity in PSAC in Zimbabwe (13). Since it is a skin condition, it is associated with psychological trauma via discrimination in its severe form. It is necessary to notify clinicians in schistosomiasis endemic areas of this finding in-order to decrease the morbidity rate associated with dermatophytosis. Further studies on this association and immunological profiling is recommended.

Children with schistosomiasis had risk reduction associated with malarial plasmodium coinfection. It is documented that malaria infection is exacerbated/ameliorated by schistosomiasis co-infection [19 - 21]. The enhanced T-helper 1 system and an increase in anti-interferon-gamma antibody causes a protective effect against the malarial parasite (32). Of note is; though schistosomiasis was demonstrated to have a protective effect with malaria in the event of co-infection malaria infection had 7-fold chance of exacerbating to complicated malaria requiring hospital admission. This makes it crucial for PSAC to be included in national schistosomiasis control programs, as there is capacity of improving the morbidity and mortality rate in children under the age of five. However, the dilemma in PSAC infections and diseases require agent attention as this is the age of growth and development during which the immune system is developing. Further studies are required to understand co-infection in PSAC inclusive of malaria infection outcome.

There was a 10 -fold chance of finding FUO and schistosomiasis as a coinfection, children with schistosomiasis had $138 \%$ risk of FUO. Despite advances in medicine, the proportion of patients discharged with undiagnosed FUO after systematic examination has not improved (33). The cause of febrile illness is not identified in approximately $9-51 \%$ of patients, this is even higher in resource limited areas endemic for childhood illnesses (34). Of the children who had FOU and schistosomiasis there was a 2-folded chance of them having serious sequelae such as seizures. Most clinician tend to think of other conditions in contrast to neglected tropical diseases when a patient presents with a fever (18). It might be 
necessary to make it a priority to screen for schistosomiasis when a child from an endemic area presents with a fever. However further immunological investigations are necessary in-order to find if the fever is due to schistosomiasis infection itself or exacerbation of a co-infection, during the early immunological responses to diseases manifestation.

In our study the odds of having URTI was $2 \%$ higher in schistosomiasis infected children with a $1 \%$ increases risk of S. haematobium infected children acquiring a URTI. Furthermore, in the coinfected cohort odds of ending up with severe pneumonia was 7-fold compared to schistosomiasis negative population. This is a very significant finding as ARIs are the leading cause of morbidity and mortality in children under the age of five in Zimbabwe (13). Thus tackling schistosomiasis crisis in this age group will have enormous contribution in reducing the mortality and morbidity rate.

The strength of this study included that although the calculated sample was 368, we managed to enrol 415 participants. We focused on the major morbidity and mortality causes in this age group from our study area which also fit into most of the low income countries were schistosomiasis is endemic. The main limitation of this study is there is a possibility that the children may have had more than two infections as co-infections which might make our data biased, however we did thorough clinical examinations and laboratory tests in all the participants to rule this out. We also considered socioeconomic status as a confounding factor by focusing on children with a similar background and in the same villages with similar lifestyles.

\section{Conclusion}

This study is novel as it demonstrates an association between schistosomiasis and top morbidity conditions in a schistosomiasis endemic area namely: URTI, dermatophytosis, malaria and FUO. Furthermore, we demonstrated a detrimental effect were coinfection led to severe sequelae of these clinical conditions. The clinical conditions described in this paper have a high impact on morbidity and mortality in children under the age of five. This brings out the importance of including PSAC in national schistosomiasis control programs. It is of paramount importance that clinician and policy makers in endemic areas are alerted of these associations in order to reach the WHO sustainable developmental goal (SDG) 3 by 2030 .

\section{Declarations}

\section{Data Availability}

The statistical data on the parasitology and clinical scores used to support the findings of this study are available from the corresponding author upon request.

\section{Conflict of Interest}

The authors declare that there is no conflict of interest.

\section{Funding Sources}

The study received funding from the TIBA. This research was commissioned by the National Institute of Health Research (NIHR), Global Health Research Programme (16/136/33) using UK aid from UK Government. The views expressed in this publication are those of the authors and not necessarily those of the NIHR or the Department of Health and Social Care.

\section{Acknowledgements}

We would like to acknowledge the Ministry of Health and Child Care, the Medical Research Council of Zimbabwe, village health workers, nursing staff, parents and children from Shamva. A special thanks to members of the Biochemistry Department at the University of Zimbabwe for technical support during field parasitology and sampling. Our most profound gratitude to the participants and their parents or guardians for taking part in this study.

\section{Author Contributions}

TLMJ, TN, ES and TM conceived and designed the study. TLMJ, TM, HM, AV, MK, EC and LJ performed the clinical examination or parasitology and the data analysis. TLMJ wrote the first draft and all authors contributed to the manuscript and revised the final version.

\section{References}

1. Mutapi F, Rujeni N, Bourke C, Mitchell K, Appleby L, Nausch N, et al. Schistosoma haematobium Treatment in 1 - 5 Year Old Children : Safety and Efficacy of the Antihelminthic Drug Praziquantel. 2011;5(5).

2. Almeida SR. Immunology of dermatophytosis. Mycopathologia. 2008;166(5-6):277-83.

Page $5 / 9$ 
3. World Health Organisation. Schistosomiasis Weekly Epidemiological report. World Heal Organ. 2016;91(49):585-600.

4. WHO. schistosomiasis [Internet]. Global Health Estimates 2015: Deaths by Cause, Age, Sex, by Country and by Region, 2000-2015. 2016. Available from: http://www.who.int/healthinfo/global_burden_disease/estimates/en/index1.html\%0AGeneva, World Health Organization; 2016.

5. Kalinda C, Chimbari MJ, Mukaratirwa S. Schistosomiasis in Zambia: a systematic review of past and present experiences. 2018;1-10.

6. Abruzzi A, Fried B. Coinfection of Schistosoma (Trematoda) with Bacteria, Protozoa and Helminths. Vol. 77, Advances in Parasitology. 2011. $1-85 \mathrm{p}$.

7. Vennervald BJ, Dunne DW. Morbidity in schistosomiasis: An update. Curr Opin Infect Dis. 2004;17(5):439-47.

8. Zhang XS, Cao KF. The impact of coinfections and their simultaneous transmission on antigenic diversity and epidemic cycling of infectious diseases. Biomed Res Int. 2014;2014:8-12.

9. Araújo MP De, Burger E, Dias Novaes R, Ami Akatuti A, Rodrigues MÂ, Mendes ACSC, et al. Impact of paracoccidioides brasiliensis coinfection on the evolution of schistosoma mansoni-induced granulomatous liver injury in Mice. Biomed Res Int. $2019 ; 2019$.

10. King, H C. parasites and poverty: the case of schistosomiasis. 2011;113(2):95-104.

11. Osakunor DNM, Woolhouse MEJ, Mutapi F. Paediatric schistosomiasis: What we know and what we need to know. PLoS Negl Trop Dis. 2018;12(2):1-16.

12. UNDP-Zimbabwe. Reduce child mortality where we are? [Internet]. 2015. Available from: https://www.zw.undp.org/content/zimbabwe/en/home/post-2015/mdgoverview/overview/mdg4.html

13. Zimbabwe national statistics survey. Demographic and health surveys.

14. Tantilipikorn P, Auewarakul P. Airway allergy and viral infection. Asian Pacific J Allergy Immunol. 2011;29(2):113-9.

15. Short S, Bashir H, Marshall P, Miller N, Olmschenk D, Prigge K, et al. Diagnosis and Treatment of Respiratory Illness in Children and Adults. Inst Clin Syst Improv [Internet]. 2017; Available from: www.icsi.org

16. Center for Disease Control and Prevention. Nonspecific Upper Respiratory Tract Infection. Cent Dis Control Prev. 2006;134(6):2001.

17. WHO. Revised WHO Classification and Treatment of Childhood Pneumonia at Health Facilities: Evidence Summaries [Internet]. World Health Organization. 2014. 26 p. Available from:

https://apps.who.int/iris/bitstream/handle/10665/137319/9789241507813_eng.pdf;jsessionid=2089DD8EDCA2FD8BFBF8678DB27578FA? sequence $=1$

18. World Health Organization. Recommendations for management of common childhood conditions. Parassitologia [Internet]. $2012 ; 84$. Available from: http://europepmc.org/abstract/MED/10697870

19. STRAUSS WG. Fever of Unknown Origin. Postgrad Med. 1964;36(1):555-9.

20. Services H, Bureau CH. Illinois Emergency Medical. 2016;

21. WHO. Giemsa Staining of Malaria Blood Films Malaria Microscopy Standard Operating Procedure-Mm-Sop-07a 1. Purpose and Scope. Malar Microsc Stand Oper Proced - MM-SOP-07A [Internet]. 2016;1-6. Available from:

http://www.wpro.who.int/mvp/lab_quality/2096_oms_gmp_sop_07a_rev.pdf

22. World Health Organization. WHO Severe malaria 2014. Trop Med Int Heal [Internet]. 2014;19 (Suppl.:7-131. Available from: http://www.who.int/malaria/publications/atoz/who-severe-malaria-tmih-supplement-2014.pdf

23. Schlenker S. Standard operating procedure. Text Chem Color. 1997;29(7):283-6.

24. Women's and Children's Hospital. Paediatric burn guidelines. Gov South Aust [Internet]. 2010;(08):8161. Available from: http://www.wch.sa.gov.au/

25. Wami WM, Nausch N, Bauer K, Midzi N, Gwisai R, Simmonds P, et al. Comparing parasitological vs serological determination of Schistosoma haematobium infection prevalence in preschool and primary school-aged children: Implications for control programmes. Parasitology. 2014;141(14):1962-70.

26. Ferreira MD, Collaniere AC, Bertolini DL, Barros NC, de Moraes Vasconcelos D. Cellular immunodeficiency related to chronic dermatophytosis in a patient with Schistosoma mansoni infection: Can schistosomiasis induce immunodeficiency? Rev Soc Bras Med Trop. 2017;50(1):1414.

27. Grappel SF, Bishop CT, Blank F. Immunology of dermatophytes and dermatophytosis. Bacteriol Rev. 1974;38(2):222-50.

28. Woodfolk JA. Allergy and dermatophytes. Clin Microbiol Rev. 2005;18(1):30-43.

29. Deen J. Encyclopedia of Malaria. Encycl Malar. 2019;1-10.

30. Stothard JR, Sousa-Figueiredo JC, Betson M, Green HK, Seto EYW, Garba A, et al. Closing the praziquantel treatment gap: New steps in epidemiological monitoring and control of schistosomiasis in African infants and preschool-aged children. Parasitology. 2011;138(12):1593-606. 
31. Osada Y, Kanazawa T. Schistosome: Its benefit and harm in patients suffering from concomitant diseases. J Biomed Biotechnol. 2011;2011(Table 1).

32. Ekpo UF, Alabi OM, Oluwole AS, Sam-Wobo SO. Schistosoma haematobium infections in preschool children from two rural communities in ljebu East, south-western Nigeria. J Helminthol. 2012;86(3):323-8.

33. Fusco FM, Pisapia R, Nardiello S, Cicala SD, Gaeta GB, Brancaccio G. Fever of unknown origin (FUO): Which are the factors influencing the final diagnosis? A 2005-2015 systematic review. BMC Infect Dis. 2019;19(1):1-11.

34. Tan Y, Liu X, Shi X. Clinical features and outcomes of patients with fever of unknown origin: A retrospective study. BMC Infect Dis. 2019;19(1):1-7.

\section{Tables}

Table 1: Clinical conditions among children aged 1-5 years in a schistosomiasis-endemic district of Zimbabwe

\begin{tabular}{|c|c|c|c|c|}
\hline \multicolumn{2}{|l|}{ Clinical Conditions } & \multicolumn{2}{|c|}{ Schistosomiasis infection Status } & \multirow{2}{*}{$\begin{array}{l}\text { Total prevalence } \\
35.1 \% \\
\end{array}$} \\
\hline & & No & Yes & \\
\hline \multirow{2}{*}{$\begin{array}{l}\text { Upper } \\
\text { Respiratory Tract Infection (URTI) }\end{array}$} & Negative & 121 & 66 & \\
\hline & positive & 149 & 80 & \\
\hline \multirow[t]{2}{*}{ Dermatophytosis } & Negative & 244 & 96 & \multirow[t]{2}{*}{$18 \%$} \\
\hline & Positive & 25 & 50 & \\
\hline \multirow{2}{*}{$\begin{array}{l}\text { Fever of Unknown Origin } \\
\text { (FOU) }\end{array}$} & Negative & 172 & 55 & \multirow[t]{2}{*}{$45 \%$} \\
\hline & Positive & 97 & 91 & \\
\hline \multirow[t]{2}{*}{ Malaria } & Negative & 220 & 121 & \multirow[t]{2}{*}{$18 \%$} \\
\hline & Positive & 50 & 25 & \\
\hline
\end{tabular}

Table 2: Crude and adjusted odds ratio of the association between Schistosomiasis infection and other clinical conditions

\begin{tabular}{l|l|l|l|}
\hline r Clinical Conditions & Crude Odds Ratio & Adjusted Odds Ratio & Risk ratio \\
\hline :r Respiratory Tract Infection & $1.98^{*}(1.66-2.48)$ & $1.22(0.80-1.87)$ & $1.01(0.84-1.2)$ \\
\hline natophytosis & $5.10 *(2.99-8.716)$ & $4.79 *(2.78-8.252)$ & $1.38 *(1.22-1.56)$ \\
\hline r of Unknown origin & $9.07 *(5.70-14.44)$ & $10.63^{*}(6.48-17.45)$ & $2.38 *(1.90-2.983$ \\
\hline ria & & & \\
\hline
\end{tabular}

*significant at $5 \%$ level of significance

Table 3: Odds ratio of having severe sequelae from co-infections with schistosomiasis in PSAC from an endemic district.

\begin{tabular}{|l|l|l|l|}
\hline $\begin{array}{l}\text { Co-infected } \\
\text { with schistosomiasis }\end{array}$ & Sequelae experienced & AOR & 95\% Confidence interval \\
\hline Upper Respiratory Tract Infections & Severe Pneumonia & $\mathbf{8 . 4 1 *}$ & 3.09 to 22.93 \\
\hline Malaria & Severe malaria & $7.09 *$ & 1.51 to 33.39 \\
\hline Dermatophytosis & Severe dermatophytosis & $20.3^{*}$ & 4.78 to 83.2 \\
\hline Fever of Unknown Origin & Seizures & $1.62 *$ & 1.56 to 4.73 \\
\hline
\end{tabular}




\section{Figures}
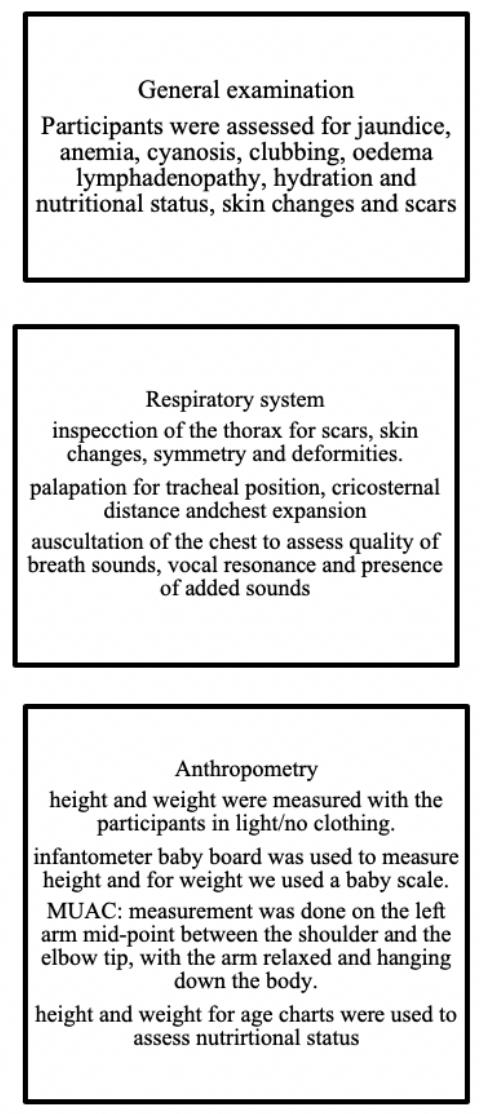

Developmental assessment

we used the childhood developmental charts from

UNICEF to measure gross motor, fine motor, language and social development
Cardiovascular system

Assess peripheral and central pulse locate the apex beat and trachea position. auscultation for heart sounds and murmurs
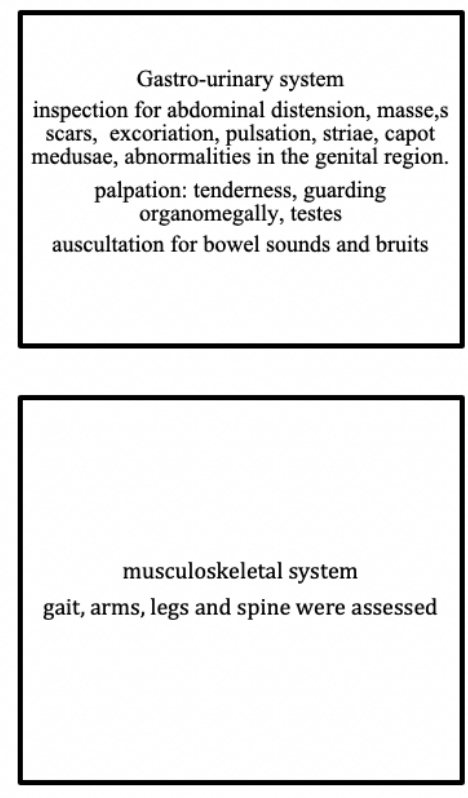

Central nervous system

we assessed the 12 cranial nerves, inspected both the upper and lower limbs for deformities, scars. tested reflexes across all joint and power across all muscle groups

\section{Figure 1}

Clinical examinations protocol 


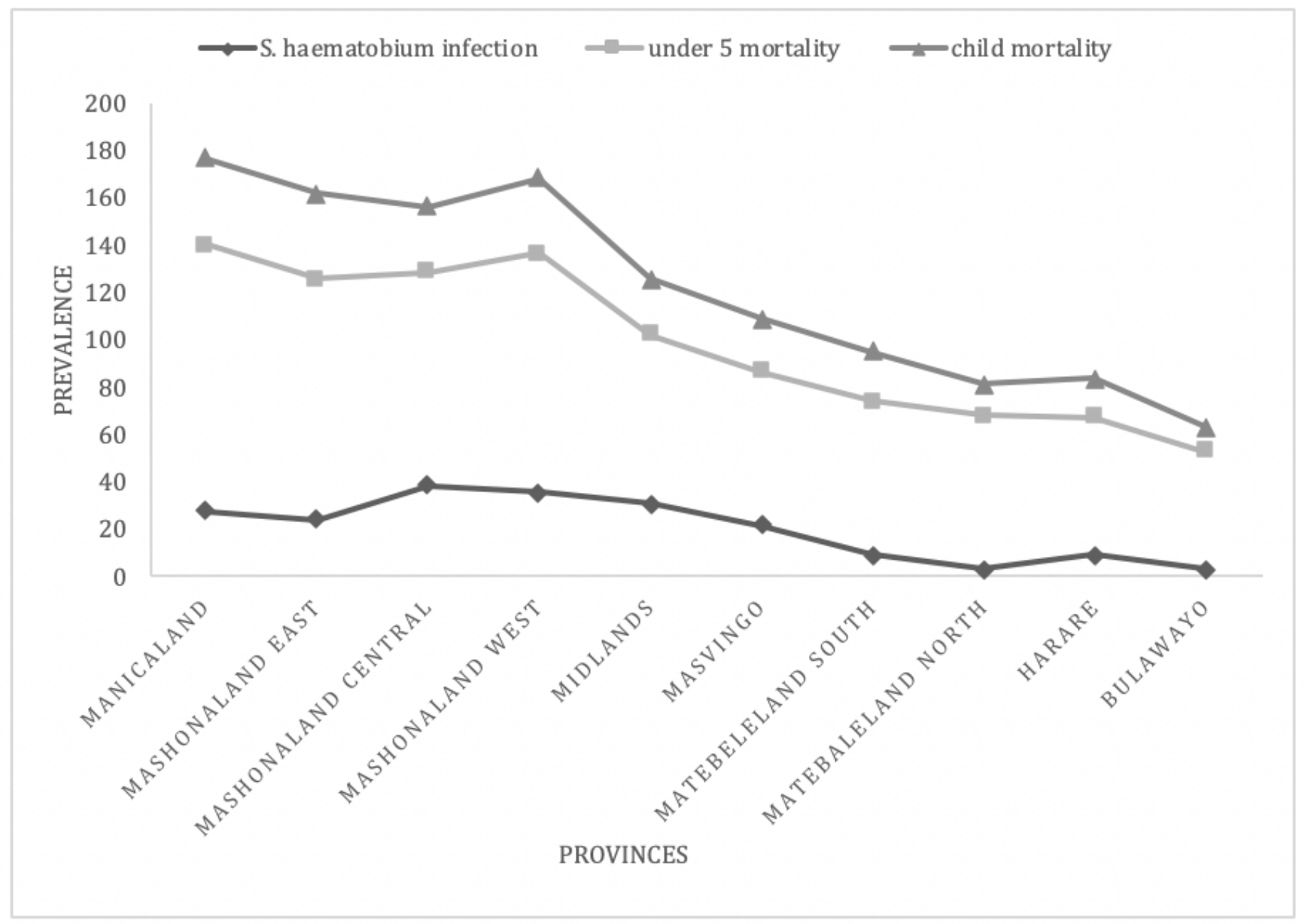

Figure 2

Trends showing schistosomiasis prevalence and under 5 child mortality per 1000 live births from Zimbabwe provinces. 